\title{
A review of the back surface metrics used as scoliosis evaluation indices
}

\author{
P Patias, Theodoros B Grivas*, A Kaspiris, C Aggouris, E Drakoutos \\ From 7th International Conference on Conservative Management of Spinal Deformities \\ Montreal, Canada. 20-22 May 2010
}

\begin{abstract}
Introduction
Although scoliosis is characterized by lateral deviation of the spine, a 3D deformation actually is responsible for geometric and morphologic changes in the trunk and rib cage. Since scoliotic deformations occur in 3D space, it is common in medical practice to use three mutually perpendicular planes to describe it: the transverse, the sagittal, and the coronal plane. In a vast related medical literature, one can find quite a few scoliosis evaluation indices, which are based on back surface data and generally measured along these planes, ie. angle of trunk rotation (ATR) or angle of trunk inclination (ATI) simulating the scoliometer measure, the Crude Trunk Asymmetry Score (CTAS), the back surface rotation (BSR) index, the volumetric asymmetry (VA), the hump severity (HS), the shoulder asymmetry index, the waist asymmetry index, the scapulae asymmetry index, the lumbar lordosis, the thoracic kyphosis, to name just a few.
\end{abstract}

\section{Materials and methods}

Regardless the large number of such indices, the literature is lacking a coherent presentation of the underlying metrics, the involved anatomic surface landmarks, the definition of planes and the related body axes definition. In addition, there exists no study of the possible correlation existing among the different indices, as well as on their meaningful clinical relevance. This paper presents a thorough study on the scoliosis evaluation indices, proposed by the medical society. In particular, the referred indices will be classified, according to the type of asymmetry they measure, according to the plane they refer to, according to the importance, relevance or the level of scientific consensus they enjoy, and according to the degree of correlation to Cobb angle.

\section{Results and discussion}

The proposed surface metrics are presented in a coherent way in an attempt to discover and analyse their possible underlying correlation mechanism. Besides the obvious aim of understanding the 3D scoliotic mechanism, an attempt is also made to organize the existing knowledge and achieve a scientific consensus on back surface scoliotic indices. It is anticipated that this will greatly help the development of future systems based on back surface measurement and reconstruction.

\section{Conclusion}

The long list of proposed scoliotic indices, which are based on surface metrics are rarely presented in crossreference to each other. This creates a possibility of misunderstandings and sometimes irrational or even wrong use of these indices by the medical society. It is hoped that the current work contributes in clearing up the issue and give rise to innovative ideas on how to assess the surface metrics in scoliosis.

Published: 10 September 2010

doi:10.1186/1748-7161-5-S1-O4

Cite this article as: Patias et al:: A review of the back surface metrics

used as scoliosis evaluation indices. Scoliosis 2010 5(Suppl 1):O4 